\title{
Experimental Stress Analysis of Fixed Partial Dentures by Use of a Dynamic Method
}

\author{
E. W. TILLITSON, R. G. CRAIG, and F. A. PEYTON \\ School of Dentistry, University of Michigan, Ann Arbor, Michigan 48104, USA
}

Using a model system replicating the size of a fixed partial denture, stress and strain analyses were made of chromium-nickel and gold alloy restorations.

Gold and chromium alloy fixed partial dentures of the maxillary posterior type have been studied with respect to surface strains using conditions of static loading. The two techniques used were the brittle lacquer ${ }^{1}$ and electronic strain gauge ${ }^{2,3}$ methods. Brumfield $^{4}$ presented work on the analysis of partial denture design using the classical beam theory. Bates ${ }^{5}$ measured dynamic strains on chromium-cobalt partial dentures using silicon transducer strain gauges and an oscilloscope.

This report describes a dynamic loading and stress analysis system; the strain as a function of time for a single impact was measured on gold and chromium alloy posterior partial dentures.

\section{Materials and Methods}

Two commonly used methods for dynamic analysis are the method in which the object under study is vibrated constantly at a single frequency or over a range of frequencies (steady state analysis) and the method in which a nondestructive impact or pulse of energy is applied to the object, thus imparting a spectrum of frequencies (transient analysis). This study used the transient method because it provided the type of loading condition usually experienced by dental structures, aside from static loads.

This investigation was supported in whole by USPHS Research Grant DE-01817 from the National Institute for Dental Research, National Institutes of Health, Bethesda, Md.

Presented in part at the 46th General Meeting of the IADR, San Francisco, Calif, March 1968.

Received for publication April 13, 1970.
An overall view of the experimental setup is shown in Figure 1. The partial denture is supported on the right by a vice that has hand-operated rotational, as well as $x-y$, motion. The partial dentures are those described in a previous study of strain using static loading. ${ }^{3}$ Just to the right of the denture is the small hammer for applying the impact. The energy of impact from the hammer may be estimated either from the angle from which it is released or from its velocity at the time of impact. In the center is a rack containing the required Wheatstone bridge-type circuitry. On the stand supporting the rack are the various power supplies, and to the left of it is the oscilloscope and camera on which results were indicated and recorded.

Figure 2 shows the gold alloy partial denture $(A)$ and the hammer $(B)$ in more detail. The hammer had hemispherical ends a half inch in diameter and had an effective mass of $28 \mathrm{gm}$.

The velocity just before impact was measured and therefore an experimentally determined calibration of the velocity and available impact energy was obtained. The calibration curve for the drop angle vs the velocity was obtained by using two solar cells $(C)$ spaced $1 \mathrm{~cm}$ apart. As the pendulum swung by the solar cells, the light beams were broken and the energy output of the cells was reduced immediately. The change in energy level was displayed as two pips on an oscilloscope trace. Knowing the sweep velocity of the oscilloscope and the distance between pips, the velocity of the hammer was calculated (kinetic energy at impact $=1 / 2 \mathrm{mv}^{2}$ ). The calibration curve is shown in Figure 3 together with the theoretical values if frictional effects are neglected; $g$ is the acceleration of gravity, 1 is the length of the pendulum, and $\theta$ is the drop angle. 


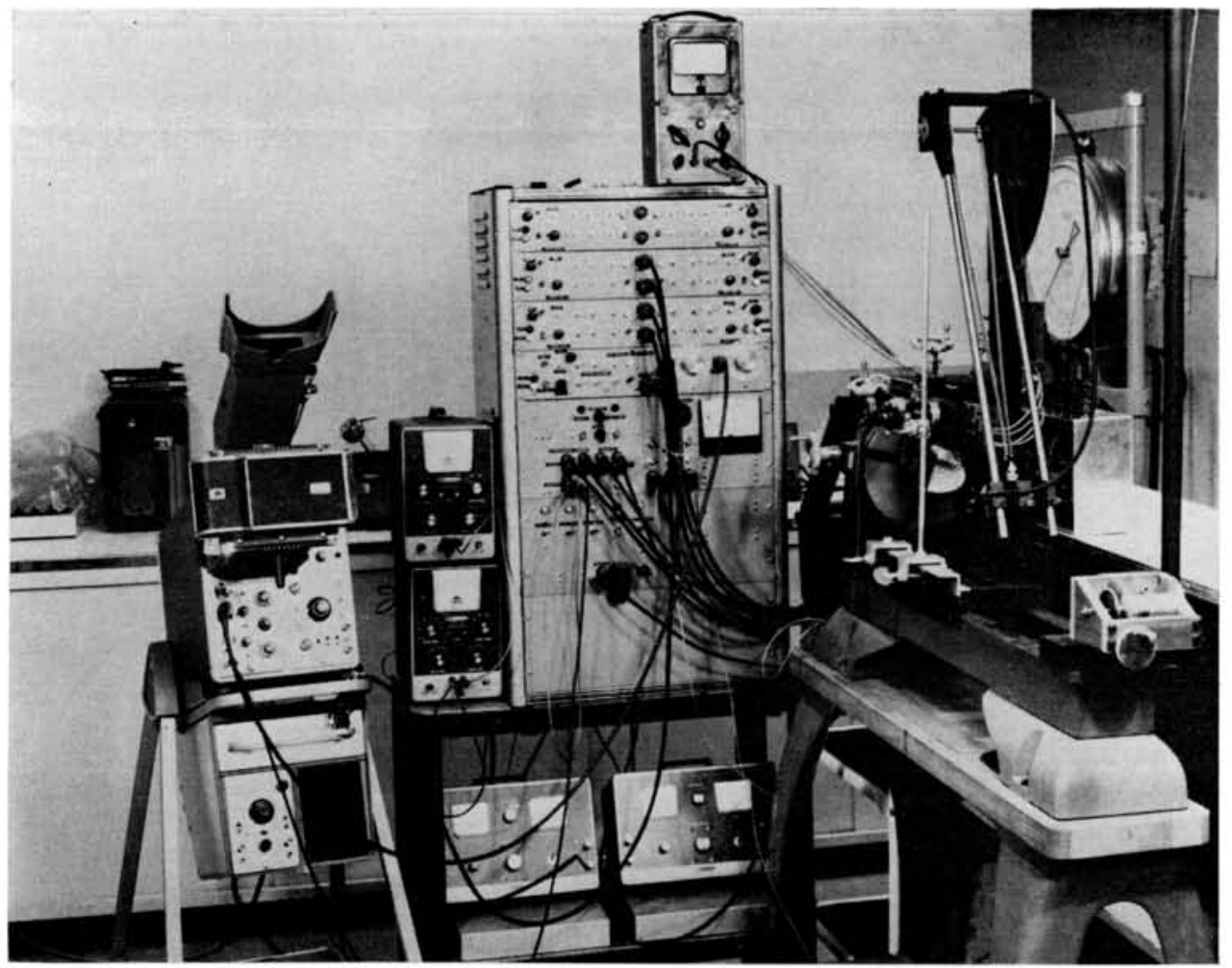

FIG 1.-Overall view of instrumentation for measuring dynamic strain of partial dentures.

Strain gauges were placed in locations where the surface was flat and thus were limited, for the most part, to the pontic facings. The orientation of the gauges was in the direction of maximum strain as determined by a previous brittle lacquer analysis of strain direction. ${ }^{1}$ The strain gauges were connected into single active arm bridge circuits. The bridges were excited from a power supply up to $6 \mathrm{v}$ dc. Each of the seven bridges was calibrated using a strain gauge load cell of known characteristics. It was found that the bridges were alike so that a single calibration curve could be used for all. No amplification of the bridge unbalance was attempted until the signal was introduced to the oscilloscope amplifier. A storage oscilloscope* and camera were used; the storage feature permitted the viewing of several repetitive traces at one time so that repeatability of an experiment could be determined. The repeata-

* Tektronix Type 564 with $3 \mathrm{~A} 3$ and $2 \mathrm{~B} 67$ plug-in units, Tektronix, Inc., Beaverton, Ore. bility usually was excellent, and when it was found to be satisfactory, the trace was photographed on high speed film. $\dagger$

To observe the beginning of the fast transients produced by this system, a scope triggering circuit was installed. This consisted of a solar cell-light beam combination that triggered the oscilloscope sweep circuit as the pendulum arm interrupted the beam immediately before the time of hammer impact. In this way the complete transient could be observed.

\section{Results}

Strain-time curves for the chromiumnickel partial denture and the gold alloy partial denture for impacts from drop angles of 45 and $30^{\circ}$ respectively are shown in Figure 4. The positions of the strain gauges are shown in the sketches; the impact was on the lingual cusp of the first molar pontic. The strain axis (vertical) was

$\dagger$ Polaroid, Type 410, Polaroid Corp., Cambridge, Mass. 


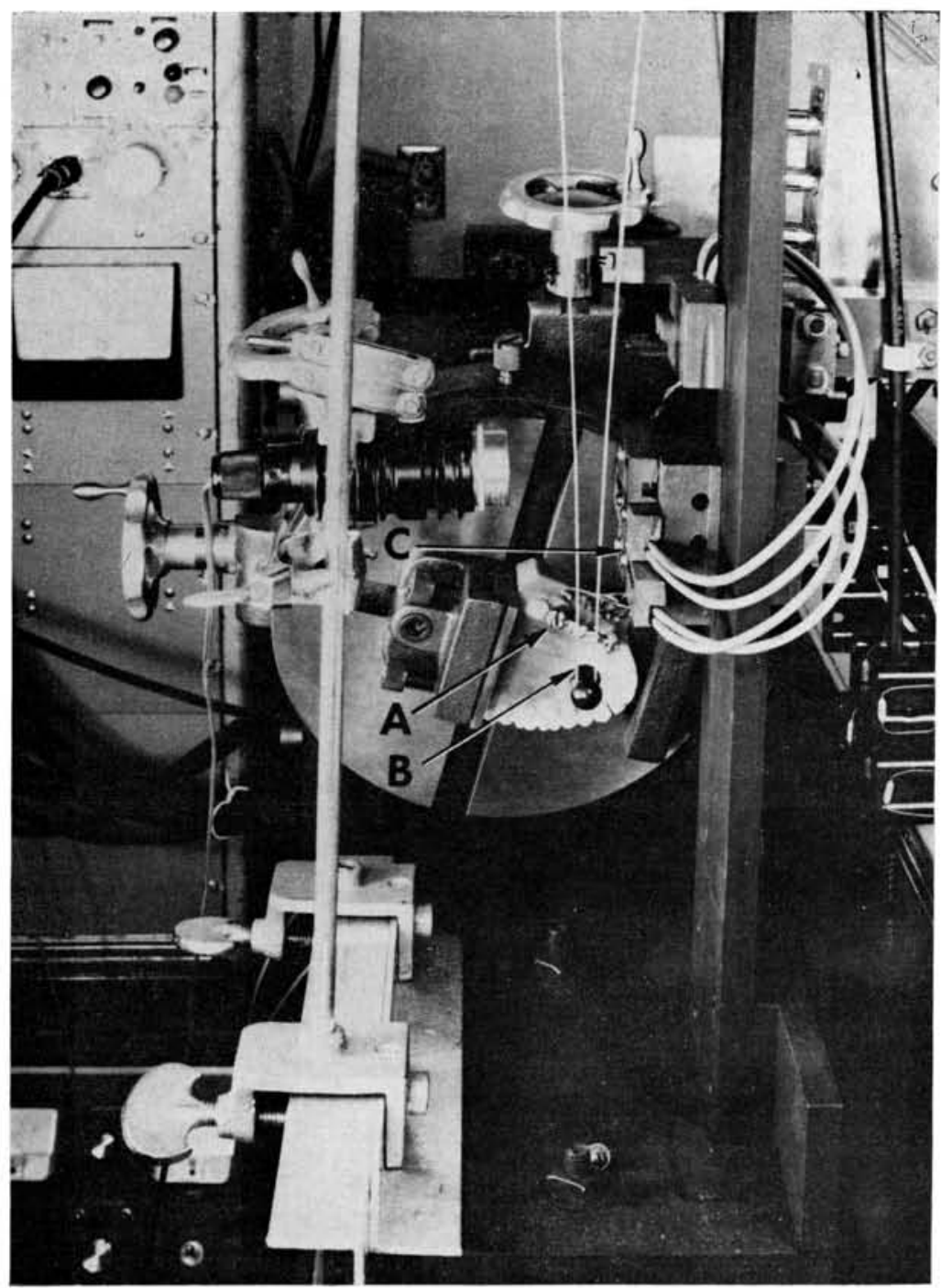

FIG 2.-Close-up showing mounted partial denture $(A)$, the hammer $(B)$, and the solar cells $(C)$.

adjusted so each $\mathrm{cm}$ (line) represented 40 uinches/inch for the chromium-nickel and $80 \mu$ inches/inch for the gold alloy denture and on the time axis (horizontal) $1 \mathrm{~cm}$ represented $0.5 \mathrm{msec}$. A downward deflection of the trace indicated compression and an upward movement showed tensile strain. The traces show that frequently the maximum strain did not occur until the denture underwent several sharp oscillations. The time for the maximum to occur was about one msec, whereas the time for the first excursion was about 20 to $30 \mu \mathrm{sec}$. Examination of the maximum amplitudes for gauges 1,2 , and 3 on the gold alloy denture showed that gauge 2, near the center of the span, exhibited the greatest strain. The maximum strains developed were 172 , 300 , and $190 \mu$ inches/inch respectively, and the corresponding stresses were 2,400 , 4,200 , and 2,660 pounds/inches ${ }^{2}$. The maximum strains on gauges 3 and 4 on the 


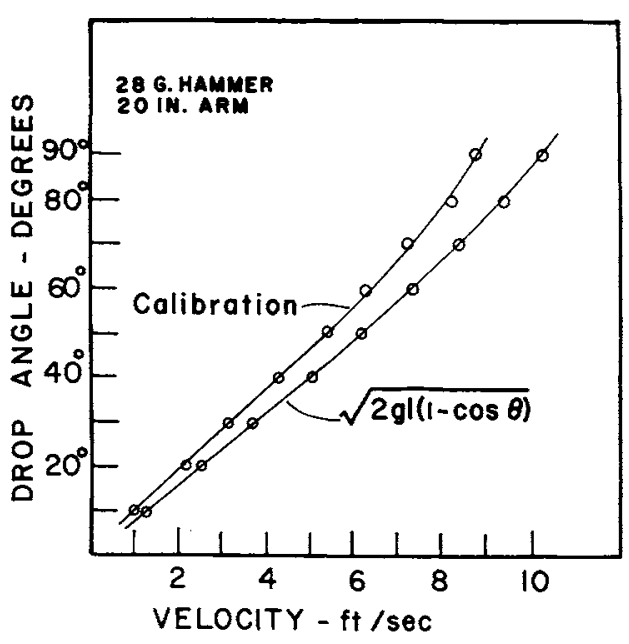

FIG 3.-Calibration and theoretical curve relating drop angle of bammer to velocity at impact.

chromium-nickel denture were 137 and 86 uinches/inch and the maximum stresses were 4,120 and 2,580 pounds/inches ${ }^{2}$.

Strain gauge 1 on the chromium-nickel denture was located on the lingual surface of the three-quarter crown on the premolar. The first strain was large and in tension and it remained in tension while the strain decayed. Gauge 4 on the chromium-nickel denture initially experienced tension followed by several compression tension cycles, but for the most part it was under tensile strain. Gauge 3 on the chromium-nickel denture first went into tension followed alternately by compression and tension.

The three gauges on the gold alloy denture first experienced compression and then tension and compression cycles. Gauge 2 gave the most symmetrical trace, whereas gauge 1 experienced generally more tension and gauge 3 more compression during the decay of strain.

It should be noted that a second contact, called contact bounce, was made between the chromium-nickel denture and the hammer at about $3 \mathrm{msec}$. This effect is shown in more detail in Figure 5 where the influence of the drop angle on the contact time and bounce is illustrated. The vertical scale is $50 \mathrm{mv} / \mathrm{cm}$; the traces top to bottom represent drop angles of $10,20,30$, and $45^{\circ}$. The time scale is $1 \mathrm{msec} / \mathrm{cm}$ except for $10^{\circ}$, which is $2 \mathrm{msec} / \mathrm{cm}$. The peak areas represent the metal hammer and the bridge in contact. Higher drop angles had slightly longer times between initial contact and bounce but the time was about $3 \mathrm{msec}$, as suggested by the strain-time curves.

The maximum stress vs the drop angle for strain gauges 2 and 3 on the chromiumnickel denture is shown in Figure 6. The impact was on the anterior buccal cusp of the first molar pontic. Such an impact caused the denture to oscillate transversely but also caused a torque so that gauge 2 always displayed an initial excursion in tension and had its maximum in tension, whereas gauge 3 displayed the opposite behavior.

The effect of impact loading on the lingual and anterior buccal cusp of the molar pontic of the gold alloy denture is shown in Figure 7. The vertical divisions represent $40 \mu$ inches/inch and the horizontal divisions $1 \mathrm{msec}$. The tracings for gauges 1 and 3 show that in addition to a transverse vibration there is also a torsional vibration. This is indicated by the transverse high frequency oscillations superimposed on lower rocking frequency vibrations. This was not observed for gauge 3 near the center of the span. It should be noted that when the impact was on the lingual cusp the initial excursion was in compression, whereas loading on the buccal cusp resulted in an initial tensile strain. Contact bounce was observed in all the lingual traces, especially on the trace for gauge 2 . As expected, the maximum strains for a $20^{\circ}$ drop angle on the lingual cusp were lower than those shown in Figure 5 for a $30^{\circ}$ drop angle.

\section{Discussion}

The dynamic method further established the nonideal behavior of even fixed posterior partial dentures. The center of the bridge functioned in a nearly ideal manner but gauges near the soldered joint between the pontics and the abutments did not. The maximum strain occurred shortly after the initial impact and was a direct function of the drop angle.

The dynamic traces showed that the dentures had both transverse and rotational modes of vibration, the latter being of lower frequency. The gold alloy and chromium- 


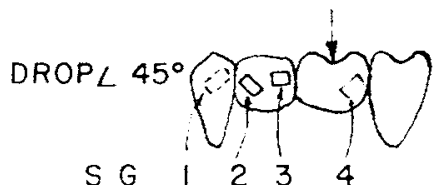

$$
\mathrm{Cr}-\mathrm{Ni}
$$



SG 1

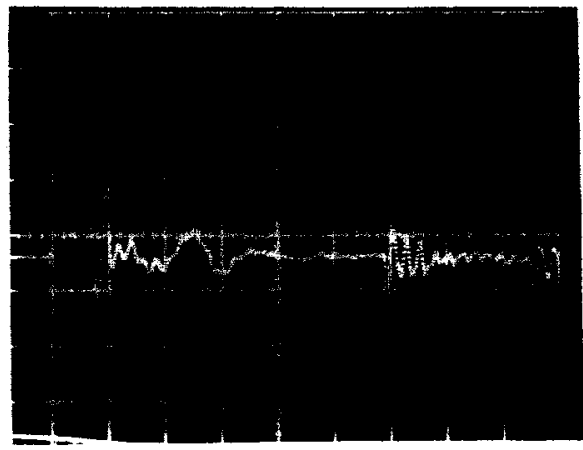

SG 3

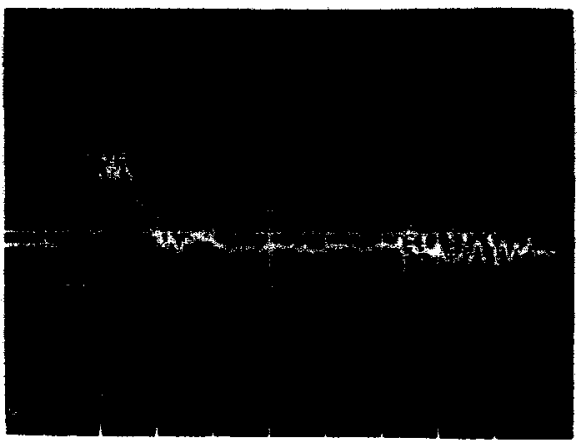

SG 4



Gold

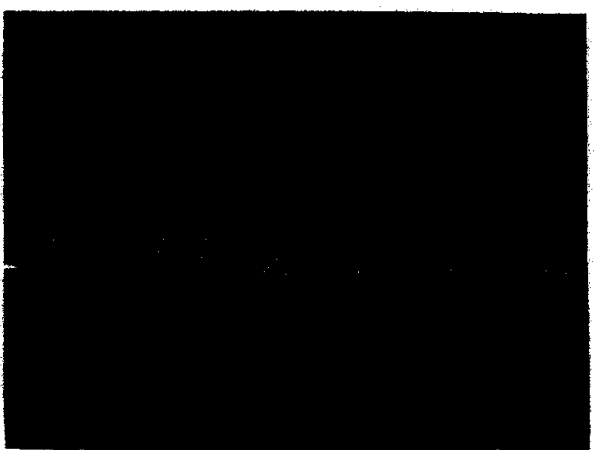

SG 1

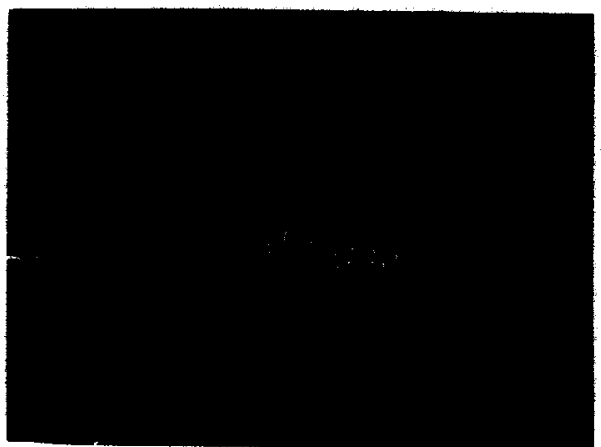

SG 2

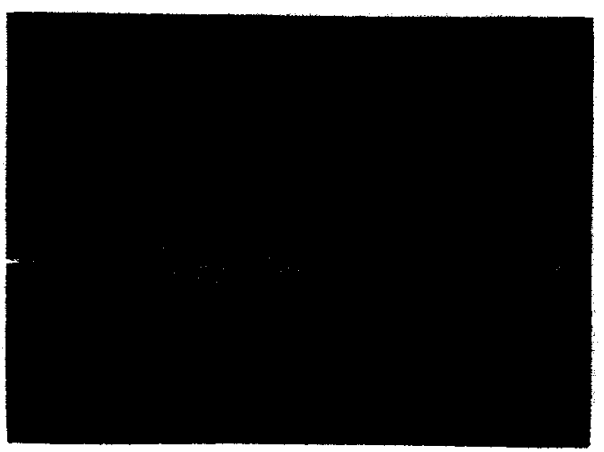

SG 3

Fig 4.-Traces for impacts on lingual cusps of first molar pontics. One vertical division represents $40 \mu$ inches/inch for the chromium-nickel and $80 \mu$ inches/inch for the gold alloy denture. 


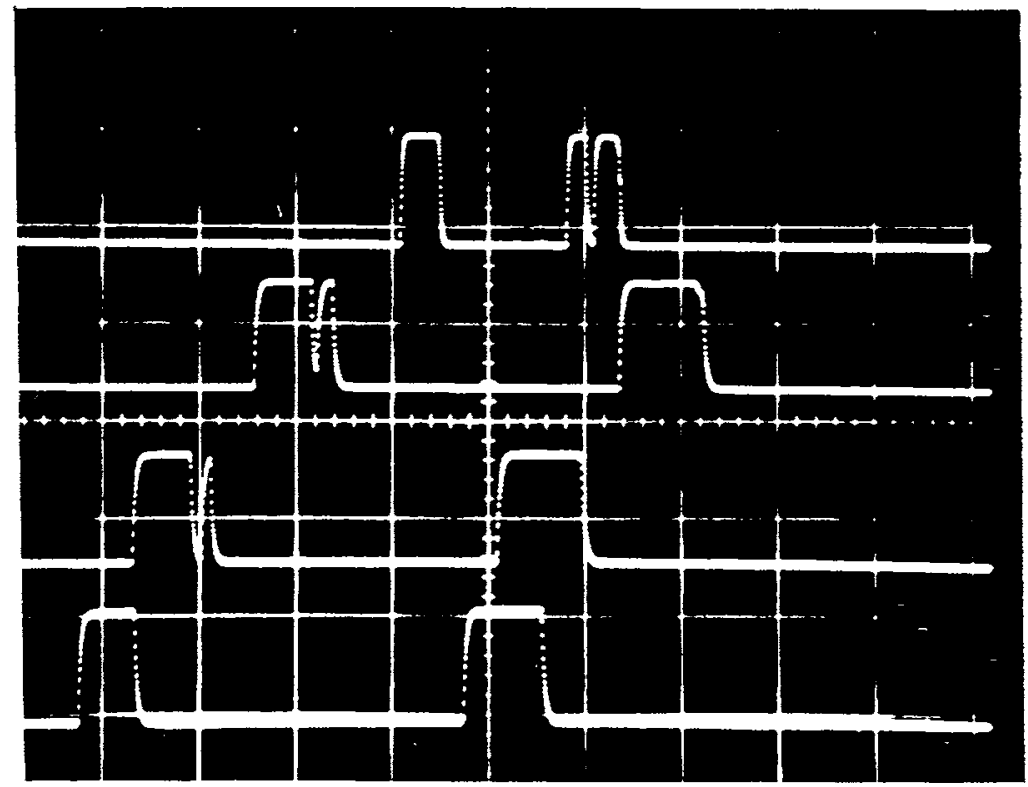

FIG 5.-Traces showing number of times bridge and hammer were in contact (peak areas) for drop angles of $10,20,30$, and $45^{\circ}$ (top to bottom).

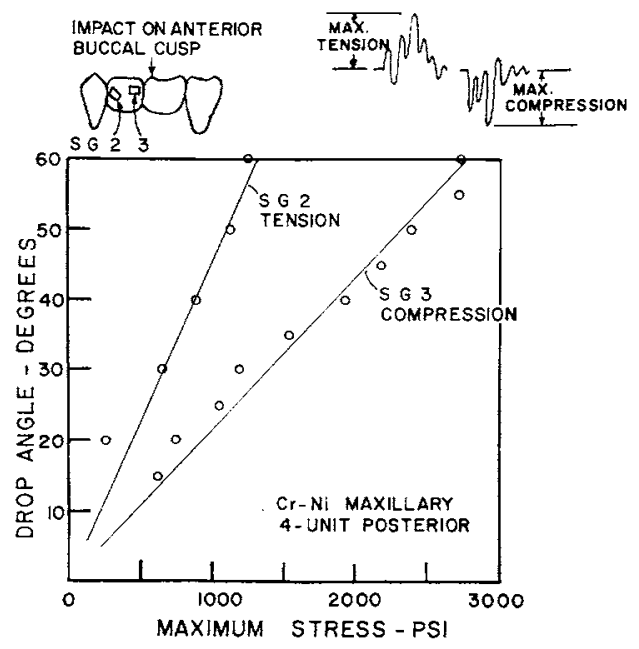

Fig 6.-Maximum stress as function of drop angle for chromium-nickel denture when impact loaded on anterior buccal cusp of molar pontic.

nickel alloy dentures had resonant frequencies of approximately 4,700 and $9,000 \mathrm{~Hz}$ respectively in the principal transverse mode. The torsional mode is a much lower frequency that has not been determined with any certainty.
Loading on the lingual and then the buccal cusp of the molar pontic reverses the type of strain on the gauges. The former gives initial tension because gauges 1 and 3 are attached near joints and loading is immediately above gauge 2 ; the latter results in initial compression because the load is on the lingual cusp and thus causes more torsion.

The contact measurements indicate that care should be taken in determining the time of the vibration for a single impact. Indications are that the vibrating denture strikes the hammer on the contact bounce rather than the reverse.

The damping-capacity coefficients were estimated by the logrithmic decrement method. For the gold alloy denture the best value was 0.12 and for the chromium-nickel bridge it was 0.21 . It should be emphasized that these measurements are on a model system and should be accepted as such.

The model system replicated the size of an actual partial denture and the roots of the typodont abutment teeth were separated from the acrylic base by a thin layer of silicone rubber simulating the periodontal ligament. The magnitudes of the strains measured, however, would not be expected 


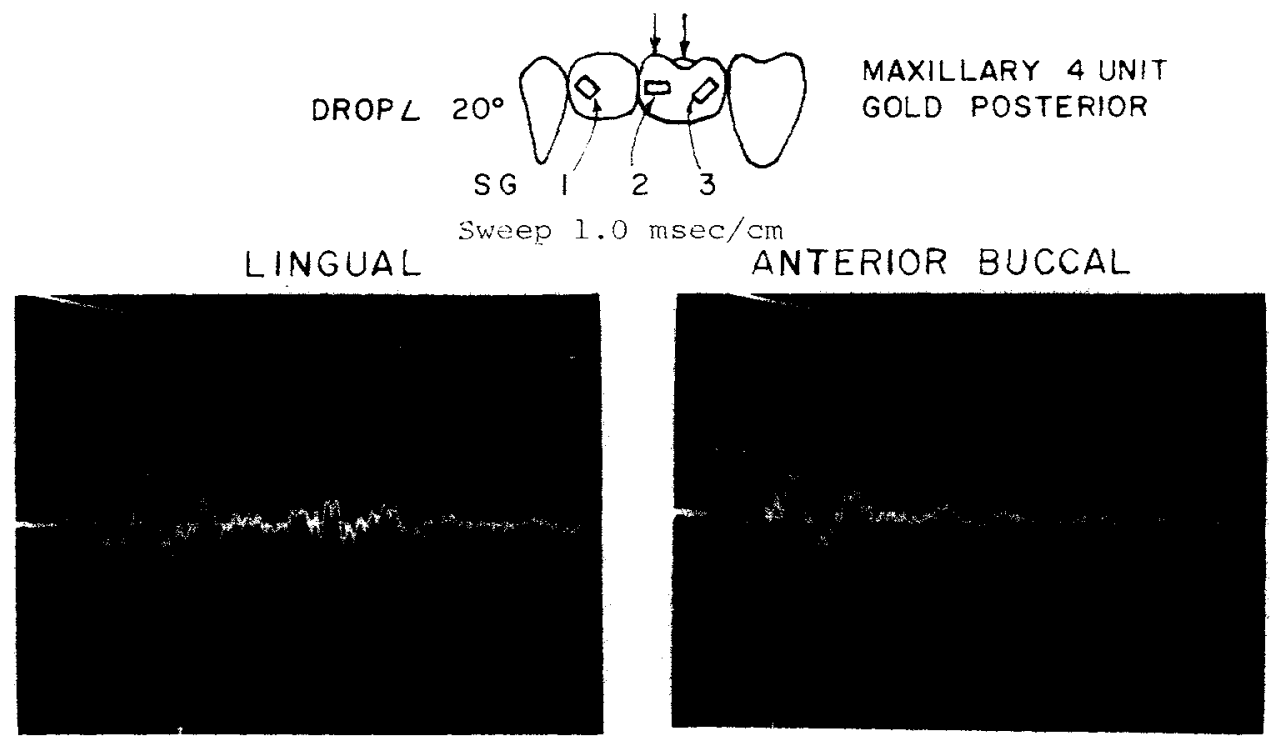

SG 1
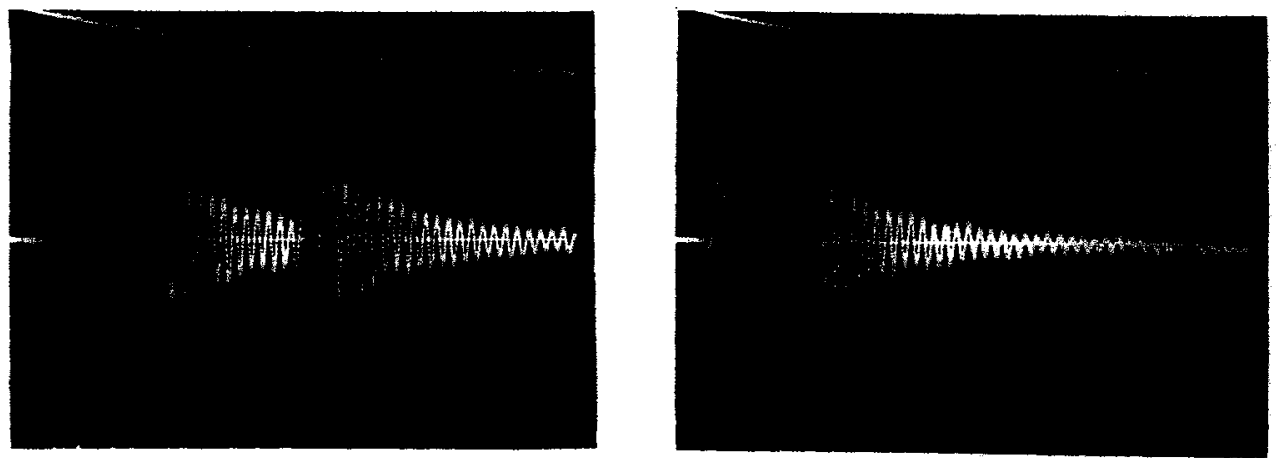

SG 2
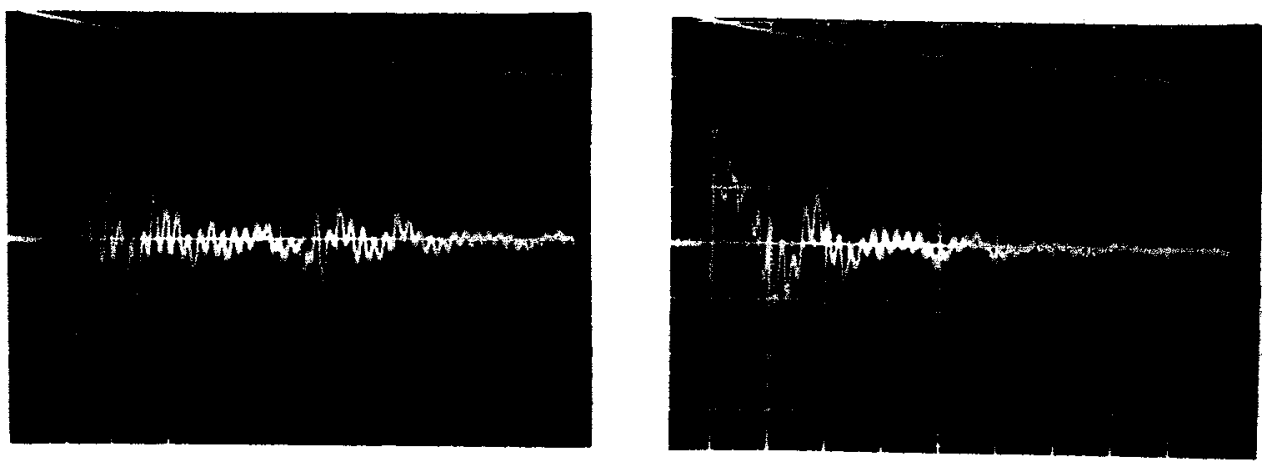

SG 3

FIG 7.-Oscilloscope traces for impacts on lingual and anterior buccal cusps of molar pontic of gold alloy denture. One vertical division represents $40 \mu$ inches/inch. 
to be identical to those in a clinical situation, but the same trends should be observed. Also, since loading was primarily point-loading, to simplify the study, the results would not represent the behavior of the denture in normal occlusion but would represent conditions when a patient bites on a small, hard object. As expected, the strains and corresponding stresses in the chromium-nickel dentures were much lower than in the gold alloy denture since the elastic moduli of the dentures are approximately $30 \times 10^{6}$ and $14 \times 10^{6}$ pounds/ inches $^{2}$, respectively. The chromium-nickel denture, therefore, was much stiffer, which was reflected by the resonant frequency of $9,000 \mathrm{~Hz}$ compared to $4,700 \mathrm{~Hz}$ for the gold alloy denture.

\section{Conclusions}

Laboratory equipment for investigating dynamic stress analysis of fixed partial dentures using a transient method was developed. The equipment was calibrated so that the strains and stresses produced, as well as their variation with time, could be estimated quantitatively.

Two maxillary four-unit posterior partial dentures have been investigated, one gold alloy and one chromium-nickel alloy. The maximum strain was a function of the energy input, but the presence of tensile or compressive strain under various conditions of loading would not have been predicted from simple beam theory. The strain-time traces always showed an oscillation of strain with time, however, at some locations and impacts, alternate compression and tension was observed, whereas under other conditions the strain was mainly tension or compression. The strain-time traces showed the dentures had transverse vibrational modes as well as torsional modes.

\section{References}

1. Craig, R.G., and Peyton, F.A.: Measurement of Stresses in Fixed Bridge Restorations, J Dent Res 44:756-762, 1965.

2. Craig, R.G., and Peyton, F.A.: Measurement of Strains in Fixed Bridges with Electronic Strain Gauges, $J$ Dent Res 46:615-619, 1967.

3. Tillitson, E.W.; Craig, R.G.; Farah, J.W.; and PEYToN, F.A.: Experimental Stress Analysis of Dental Restorations: VIII. Surface Strains on Gold and Chromium Fixed Partial Dentures, J Pros Dent 24:174-180, 1970.

4. BRUMFIELD, R.C.: Fundamental Mechanics of Dental Bridges and Structural Investigation and Design of Dental Bridges, in TYlman, S.D.: Theory and Practice of Crown and Bridge Prosthodontics, 5th ed, St. Louis: C. V. Mosby Co., 1965, pp 11181196.

5. BateS, J.F.: Studies Related to the Fracture of Partial Dentures: The Functional Strain in Colbalt-Chromium Dentures-A Preliminary Report, Brit Dent J 120:79-83, 1966. 